\title{
A Novel Device for Closed Reduction and Percutaneous Fixation of Thoracolumbar Fractures
}

\author{
CHRISTOPH LINHART ${ }^{1}$, CHRISTOPHER A. BECKER ${ }^{1}$, NIMA BEFRUI ${ }^{1}$, EDUARDO M. SUERO ${ }^{1}$, \\ ADRIAN C. KUSSMAUL ${ }^{1}$, WOLFGANG BÖCKER ${ }^{1}$, CHRISTIAN KAMMERLANDER $^{1,2}$ and AXEL GREINER ${ }^{1}$ \\ ${ }^{1}$ Department of Orthopaedics and Trauma Surgery, Musculoskeletal \\ University Center Munich (MUM), University Hospital, LMU Munich, Munich, Germany; \\ ${ }^{2}$ AUVA Traumahospital Styria, Graz, Austria
}

\begin{abstract}
Background/Aim: Open surgical reduction/fixation of thoracolumbar fractures results in significant soft-tissue trauma and related complications. Minimally-invasive technical developments could deliver similar radiological outcomes, while avoiding the related complications. We evaluated radiological and perioperative outcomes in thoracolumbar fractures by using a novel minimally-invasive device. Patients and Methods: Twenty-six patients with 29 thoracolumbar fractures using the NForce device were analyzed. Postoperative reduction and alignment were assessed by radiographic measurement of the local kyphosis angle (LKA) up until a follow-up period of 9 months. Results. Postoperative imaging revealed an average reduction of traumatic kyphosis of $8.25^{\circ}\left( \pm 7.72^{\circ}\right)$ with an average postoperative LKA of $3.24^{\circ}\left( \pm 8.97^{\circ}\right)$. The highest degree of reduction was $27.39^{\circ}$. The mean LKA had increased to $5.08^{\circ}$ $\left( \pm 5.17^{\circ}\right)$ at 3 months postoperative, $5.43^{\circ}\left( \pm 4.32^{\circ}\right)$ at 6 months and $6.21^{\circ}\left( \pm 3.82^{\circ}\right)$ at 9 months. Conclusion: The minimally invasive NForce system is effective in performing anatomic percutaneous reduction/fixation.
\end{abstract}

Although fractures of the thoracolumbar junction are a common occurrence following traumatic injuries of the spine, the optimal treatment option remains a topic of debate (1-3). Over the last few years, methods and systems

This article is freely accessible online.

Correspondence to: Christoph Linhart, MD, Department of Orthopaedics and Trauma Surgery, Musculoskeletal University Center Munich (MUM), University Hospital, LMU Munich, Marchioninistr. 15,81377 Munich, Germany. E-mail: christoph.linhart@med.unimuenchen.de

Key Words: Spine, fracture, lumbar spine, thoraco-lumbar fracture, stabilization, reduction, closed reduction, surgical treatment, Nforce, dorsal instrumentation. for minimally invasive fixation have seen a surge in the field of spinal surgery. These techniques promise faster rehabilitation, lower rates of infection and intraoperative blood loss, as well as faster postoperative mobilization due to lower pain compared to their traditional open surgical counterparts (4-7). Given that closed reduction of complex fractures is not always possible, traditional open surgical methods remain an essential part of the trauma surgeon's standard repertoire.

Anatomic fracture reduction of thoracolumbar fractures is essential in restoring the natural alignment of the spine. Closed fracture reduction, which can be performed on the spinal column by positioning manoeuvres, can achieve good corrections of the alignment of the spine (2). By combining these positioning manoeuvres with a prebending of the spinal rods in percutaneous minimally invasive dorsal instrumentation of thoracolumbar and lumbar fractures, Tinelli et al. were able to achieve a good realignment of the spinal column fractures (8). When used for extended reduction and spinal realignment, however, this method increases the risk of pedicle screw cut-out. While the open dorsal approach has the advantage to use monoaxial pedicle screws and thus offer the possibility to improve reduction with manually applied compression/distraction/lift of the fractured vertebrae, percutaneous systems most often use polyaxial pedicle screws and, therefore, lack this advantage $(3,6)$. Open dorsal spondylodesis, however, has considerable drawbacks compared to percutaneous dorsal instrumentation, as mentioned above $(4,5,9)$.

Recently, a novel reduction tool for minimally invasive percutaneous posterior instrumentation (NForce, Medtronic plc, Minneapolis, USA) has been introduced. The device was bale to improve minimally invasive reduction of spine fractures and, thus, achieve a better reduction result. In the current study, we describe the technical application of the NForce system and present clinical results of a group of 26 patients treated with the device. 


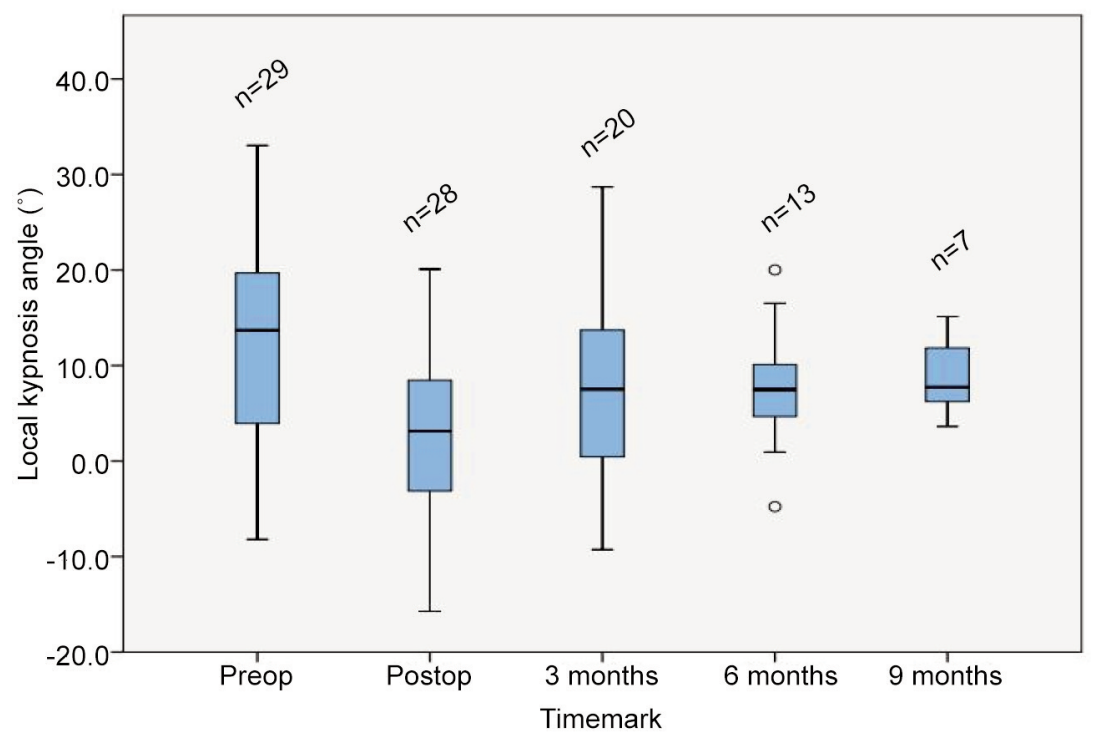

Figure 1. Boxplot representation of local kyphosis angles measured at the following timemarks: preoperative, postoperative, 3 months post-op, 6 months post-op and 9 months post-op. $n$ indicates the number of vertebrae analyzed at the given timemark. Note that negative LKA values indicate a local lordosis/reclination.

\section{Patients and Methods}

This study was approved by the Ethics Committee of our Institution with the approval number 18-030. Patients who were treated for thoracolumbar fractures using the NForce system between February 2017 and June 2019 were included in our study. The inclusion criteria were: 1) traumatic fracture of the thoraco-lumbar spine AO Type A or B without neurological deficit and 2) fixation of the fracture using the NForce instrumentation. Exclusion criteria were 1) non-traumatic spinal fracture (e.g., pathologic fractures) und 2) concomitant cervical spine fracture. Fractures were classified using the AO nomenclature. All procedures were performed using percutaneous dorsal instrumentation and reduction with positioning manoeuvres. A total of 26 patients met criteria and were included in the study. Out of the 26 patients, 2 presented fractures at multiple levels of the spinal column, resulting in a total of 29 fractured vertebrae assessed postoperatively. The angle measurement results were summarized in Figure 1 and Table I.

The NForce system is designed for performing closed reduction of spinal fractures in combination with the minimally invasive Medtronic CD HORIZON LONGITUDE II System (Medtronic plc, Minneapolis, MN, USA). To perform the reduction, sleeves of the NForce device are mounted and fixed to the pedicle screw sleeves. The system is then assembled including a double-ratchet mechanism. This double system, mounted in bilateral fashion, provides the ability of performing multiplanar reduction manoeuvres, e.g., kyphosis, lordosis, distraction and compression. The amount of correction can be actively controlled in real time using fluoroscopy. Particular attention should be paid on potential induced scoliosis following the closed reduction manoeuvres. While final reduction is assessed on the discretion of the responsible consultant, we aimed for a complete reduction whenever possible. Following the reduction manoeuvre, the longitudinal rod is inserted in an appropriate length and bend. To illustrate the effect of the angle changes pre- to postoperatively we refer to the radiological imaging which were included in Figure 2. The intraoperative application is also shown in Figure 2.

The primary outcome measure was spinal reduction and alignment, as assessed by measuring the local kyphosis using the bisegmental Cobb-angle in lateral spinal radiography in all patients, additionally in EOS or sagittal CT-scan. The Cobb angle was measured in images performed preoperatively, postoperatively and at routine follow-up visits conducted at 3 months, 6 and 9 months postoperatively. Follow-up imaging data was available for 19 patients after 3 months, 13 patients after 6 months, and 7 patients after 9 months. Secondary outcome measures included: duration of surgery, intraoperative blood loss and days of hospitalization/days of inpatient treatment. Descriptive statistics were calculated for each variable. Pairwise comparisons were conducted using the Wilcoxon signed-rank test, with $\alpha$ set to 0.05 . All analyses were conducted using SPSS (Version 25, IBM, Armonk, NY, USA).

\section{Results}

A total of 19 male and 7 female patients, with ages ranging from 18 to 87 years (mean age: $55 \pm 22.5$ years), were included in the study. Fractures situated in the thoracolumbar junction were found to be the most prevalent, with fractures of L1 being the most common $(24.1 \%$; $n=7)$, followed by fractures of T12 $(13.8 \% ; n=4)$. All basic data are shown in Table II.

Fracture reduction and postoperative alignment. Vertebral fractures were shown to cause drastic changes in the spinal curvature, with preoperative local kyphosis angle (LKA) values ranging from $33.04^{\circ}$ to $-8.21^{\circ}$ (negative LKA values 
Table I. Preoperative and postoperative angle measurement of spinal colum curvature.

\begin{tabular}{|c|c|c|c|c|}
\hline Case no. & $\begin{array}{c}\text { Fracture } \\
\text { level }\end{array}$ & $\begin{array}{c}\text { Preop } \\
\text { LKA }\left(\left(^{\circ}\right)\right.\end{array}$ & $\begin{array}{l}\text { Postop } \\
\text { LKA }\left(\left(^{\circ}\right)\right.\end{array}$ & $\begin{array}{c}\text { Postop } \\
\Delta\left(\left(^{\circ}\right)\right.\end{array}$ \\
\hline 1 & L2 & 0.75 & -3.72 & -4.47 \\
\hline 2 & $\mathrm{~T} 12$ & 15.09 & 14.03 & -1.06 \\
\hline 3 & T9 & 21.26 & 6.39 & -14.87 \\
\hline 4 & $\mathrm{~T} 8$ & 19.09 & 5.95 & -13.14 \\
\hline 5 & $\mathrm{~T} 4$ & 7.28 & 2.01 & -5.27 \\
\hline 6 & $\mathrm{~L} 2$ & 2.50 & -15.73 & -18.23 \\
\hline 7 & L1 & 6.86 & 3.14 & -3.72 \\
\hline 8 & L1 & 4.19 & 4.01 & -0.18 \\
\hline 9 & L1 & 29.58 & 2.19 & -27.39 \\
\hline 10 & L1 & 9.34 & -2.54 & -11.88 \\
\hline 11 & L1 & 11.45 & -9.95 & -21.4 \\
\hline 12 & $\mathrm{~L} 2$ & 3.93 & -8.74 & -12.67 \\
\hline 13 & L1 & 7.78 & 3.09 & -4.69 \\
\hline 14 & L1 & -5.23 & -8.45 & -3.22 \\
\hline \multirow[t]{3}{*}{15} & $\mathrm{~T} 5$ & 24.37 & 16.84 & -7.53 \\
\hline & $\mathrm{T} 7$ & 19.69 & 7.93 & -11.76 \\
\hline & T9 & 15.00 & 13.20 & -1.80 \\
\hline 16 & $\mathrm{~T} 8$ & -3.55 & -3.91 & -0.36 \\
\hline \multirow[t]{2}{*}{17} & $\mathrm{~T} 10$ & -8.21 & -8.13 & 0.08 \\
\hline & $\mathrm{T} 11$ & 0.73 & 0.73 & 0.00 \\
\hline 18 & $\mathrm{~T} 12$ & 29.45 & 4.75 & -24.7 \\
\hline 19 & $\mathrm{~T} 11$ & 19.50 & 8.98 & -10.52 \\
\hline 20 & $\mathrm{~T} 11$ & 17.54 & 6.89 & -10.65 \\
\hline 21 & $\mathrm{~T} 10$ & -2.40 & -1.67 & 0.73 \\
\hline 22 & $\mathrm{~T} 12$ & 13.69 & 3.11 & -10.58 \\
\hline 23 & $\mathrm{~T} 12$ & 15.42 & 13.59 & -1.83 \\
\hline 24 & $\mathrm{~T} 6$ & 24.56 & 20.09 & -4.47 \\
\hline 25 & $\mathrm{~T} 7$ & 20.51 & 16.72 & -3.79 \\
\hline 26 & $\mathrm{~T} 7$ & 33.04 & I & I \\
\hline
\end{tabular}

Preop. and postop LKA (local kyphosis angle) indicate local kyphosis angle (a.k.a. Bisegmental Cobb-angle). Postop. relative angle quantifies the angular increase achieved by fracture reduction using the NForce system. Negative values indicate lordosis/reclination. L: Lumbar vertebrae; $\mathrm{T}$ : thoracic vertebrae.

indicate local lordosis). The mean preoperative LKA was $12.18^{\circ}\left( \pm 11.10^{\circ}\right)$. By the use of the N-Force system, an average reduction of traumatic kyphosis of $8.25^{\circ}\left( \pm 7.72^{\circ}\right)$ was achieved, as determined in postoperative radiography or CT-scan. This led to a mean postoperative LKA of $3.24^{\circ}$ $\left( \pm 8.97^{\circ}\right)$, indicating a nearly neutral LKA. The greatest reduction of traumatic local kyphosis performed using the $\mathrm{N}$ Force system was $27.39^{\circ}$.

At the 3-month mark, postoperative LKA values had increased by a mean of $5.08^{\circ}\left( \pm 5.17^{\circ}\right)$. The mean LKA at 3 months post-op was $7.02^{\circ}\left( \pm 9.73^{\circ}\right)$. After 6 months postoperative, LKA values had increased by a mean of $5.43^{\circ}$ $\left( \pm 4.32^{\circ}\right)$ compared to immediate post-op imaging, resulting in a mean LKA of $7.78^{\circ}\left( \pm 6.61^{\circ}\right)$. In 7 patients, spinal imaging data performed at 9 months post-op was available (follow-up rate: $26.9 \%$ ). After 9 months postoperative, LKA
Table II. Clinical characteristics of included patients.

\begin{tabular}{lc}
\hline Parameter & Value \\
\hline Gender (no) & \\
Male & 19 \\
Female & 7 \\
Age (years) & $55 \pm 22.5$ \\
Blood loss (ml) & \\
2 segments $(\mathrm{n}=17)$ & $126.5 \pm 91.2$ \\
3 segments $(\mathrm{n}=2)$ & $200 \pm 0$ \\
4 segments $(\mathrm{n}=5)$ & $206 \pm 124.2$ \\
8 segments $(\mathrm{n}=1)$ & 200 \\
Operating time (min) & \\
2 segments $(\mathrm{n}=17)$ & $113 \pm 53$ \\
3 segments $(\mathrm{n}=2)$ & $163 \pm 28$ \\
4 segments $(\mathrm{n}=5)$ & $150 \pm 27$ \\
8 segments $(\mathrm{n}=1)$ & 312 \\
Fracture localisation [no (\%)] & \\
T4 & $1(3.4 \%)$ \\
T5 & $1(3.4 \%)$ \\
T6 & $1(3.4 \%)$ \\
T7 & $3(10.3 \%)$ \\
T8 & $2(6.9 \%)$ \\
T9 & $2(6.9 \%)$ \\
T10 & $2(6.9 \%)$ \\
T11 & $3(10.3 \%)$ \\
T12 & $4(13.8 \%)$ \\
L1 & $7(24.1 \%)$ \\
L2 & $3(10.3 \%)$ \\
cases requiring anterior interbody & $4(15.4 \%)$ \\
cage implantation [no (\%)] & \\
\hline &
\end{tabular}

values had increased by $6.21^{\circ}\left( \pm 3.82^{\circ}\right)$ compared to immediate post-op imaging. The mean LKA at 9 months post-op was $8.94^{\circ}\left( \pm 4.30^{\circ}\right)$ shown in Figure 3.

Secondary outcomes. The mean duration of surgery was 113 $( \pm 53)$ minutes. There was a mean intraoperative blood loss of $126( \pm 81) \mathrm{ml}$, with a maximum of $300 \mathrm{ml}$. In cases requiring dorsal stabilization across $3(n=2)$ or $4(n=5)$ segments, the mean operating time was $163( \pm 28)$ minutes and $150( \pm 27)$ minutes, respectively, with a mean intraoperative blood loss of $200 \mathrm{ml}$ for 3 segment stabilization and $206 \mathrm{ml}$ for 4 segment stabilization. In a single case, dorsal stabilization spanning across 8 segments was necessary due to fractures at 3 different levels of the spinal column, resulting in a duration of surgery of $312 \mathrm{~min}$ and a blood loss of $200 \mathrm{ml}$.

Complications were encountered in two cases. In one case involving fracture reduction by $18.23^{\circ}$, a vertical connecting rod cut out from the pedicle screw interface postoperatively, therefore requiring corrective surgery. In a second patient, a postoperative collapse of the spinal column, which led to a local kyphosis angle of $28.96^{\circ}$ (post-op LKA: $14.03^{\circ}$ ), was observed 3 months after surgery, therefore requiring 

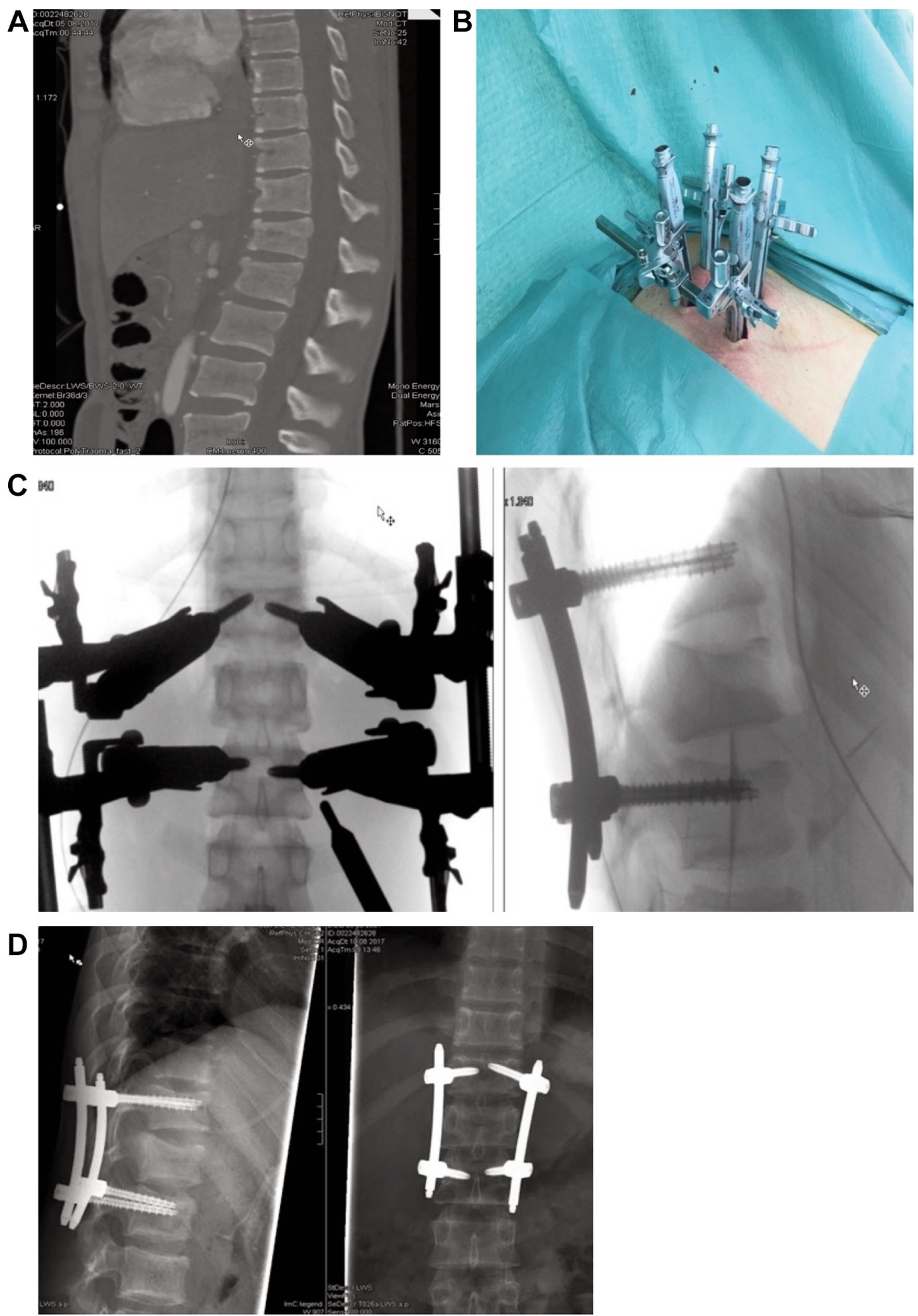

Figure 2. Images from Pat. \#1 a 2001 born male. (A) Shows a fracture of the L1 vertebral body. (B) Intraoperative use of the NForce-System: Reduction device for minimally invasive percutaneous posterior instrumentation. (C) Intraoperative fluoroscopy imaging of application of the NForce-Device. The amount of correction is reached using the double ratch mechanism. (D) x-ray one day after surgery. 


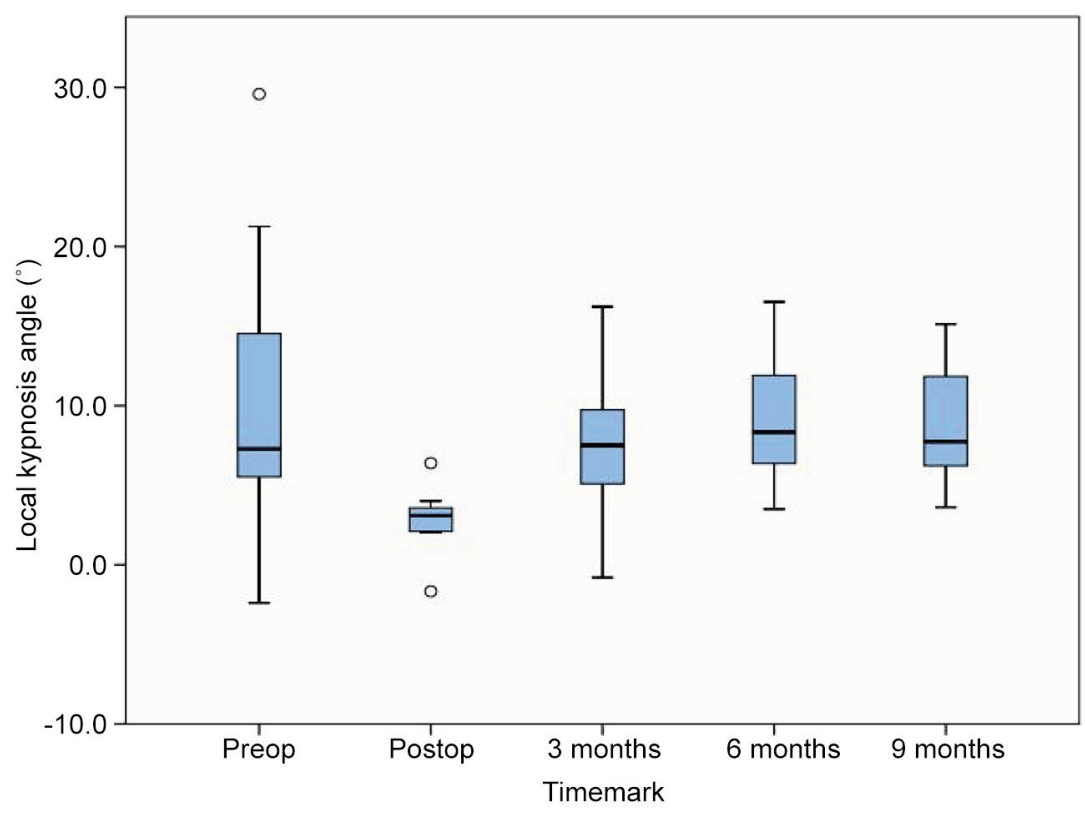

Figure 3. Boxplot representation of local kyphosis angles measured in 7 cases with spinal imaging data available up until the 9-month mark.

corrective surgery involving extended dorsal stabilization. In 4 cases, spinal fusion using anterior interbody cage implantation was performed after initial dorsal stabilization and were, therefore, excluded from further postoperative angle assessment. In 15 of 26 cases screw removal was performed after 1 year once fusion had been confirmed. Further 5 cases could be diagnosed with fusion in the available imaging. No screw loosening or screw pull-out was observed in follow-up imaging.

\section{Discussion}

Over the last decades, the surgical field has seen a transition from open to minimally invasive surgical procedures due to their demonstrated benefits of lower intraoperative blood loss, shorter hospital stay, lower reported pain levels and faster mobilisation (10-13). While many medical fields including general and thoracic surgery, urology, gynaecology and orthopaedic and trauma surgery of the extremities have now adopted a multitude of minimally-invasive surgical techniques the reduction of spinal fractures typically required open surgical approaches involving extensive operative trauma. The NForce system promises to fill this gap by enabling the percutaneous reduction and posterior instrumentation of spinal fractures. In the current study, we performed radiographic analysis of cases, in which a reduction of spinal fractures was performed using the NForce reduction tool.

We found that the postoperative status of spinal alignment and local kyphosis determined after percutaneous spinal fracture reduction mirrored that of traditional open reduction in posterior instrumentation. In our study, immediate postoperative radiographic imaging of the spine determined an average LKA of $3.24^{\circ}\left( \pm 8.97^{\circ}\right)$, with an average reduction of traumatic kyphosis of $8.25^{\circ}\left( \pm 7.72^{\circ}\right)$. A systematic review of postoperative outcomes following thoracolumbar fracture stabilization, performed by Verlaan et al., determined an identical average Cobb angle of $1.1^{\circ}$ after traditional open reduction and short posterior instrumentation, with a slightly higher average angular correction of $12.8^{\circ}$ (14).

Postoperative follow-up control showed a slight progression of local kyphosis of up to 3 degrees at 9 months postoperative, on average. This compares favourably with previous research showing an average correction loss of $7.6^{\circ}$ 12 months or more after traditional open reduction in short posterior segmentation. Long posterior segmentation (more than 2 segments) and combined anterior and posterior stabilization techniques converged at an average loss of correction of $10^{\circ}(14)$. In one case observed in our study, considerable postoperative loss of correction and increase of local kyphosis after NForce reduction and short segment posterior stabilization required corrective surgery by means of extended posterior stabilization.

These findings demonstrate that nearly identical results, concerning the amount of angular correction and postoperative stability, can be achieved by using a percutaneous reduction method. However, when comparing the postoperative stability of different reduction techniques and stabilization systems, the differences in components and technical configurations must 
also be taken into account. One example is the smaller diameter of the $5.5 \mathrm{~mm}$ connecting rods of the Longitude II system that are used in conjunction with the NForce reduction tool, compared to the $6 \mathrm{~mm}$ rods used in systems such as the Universal Spine System (USS, Synthes, PA, USA).

A clear advantage of the NForce system is the possibility of minimally invasive application. Due to the relatively complex design of the NForce reduction device compared to open monoaxial fracture treatment, the time required for surgical treatment is noticeably increased, decreases again on the other side through experience with the device. Furthermore, it is difficult to estimate a defined force and in the area of the lordotic lumbar spine, it is easy to reach the limits of the repositionability due to interfering collision of the system components. In addition to clinical studies, experimental set-ups with biomechanical stability tests on finite elements should also play a role in order to better understand the forces acting through the repositioning manoeuvre by using a new reduction system on the spine (15). Another disadvantage is that the NForce system cannot be combined with cement augmentable screws to achieve a better force distribution of the osteosynthesis on the vertebral body. However, further development of the NForce system may be able to overcome these disadvantages in the future.

Although clinical patient data, such as the intensity of pain experienced by patients, mobility, duration of hospital stay after surgery involving fracture reduction using the NForce system have yet to be investigated, such clinical benefits may be expected when choosing a less invasive and traumatic surgical method. Despite the limited case number and timeframe of our study, radiographic results following percutaneous spinal fracture reduction proved to be promising and on par with those observed after traditional open fracture reduction.

\section{Conclusion}

Our results show that the minimally-invasive NForce system is effective in performing percutaneous reduction of thoracolumbar spinal fractures in achieving angular corrections of spinal curvature and postoperative stability. The system offers an alternative to open surgical approaches that could reduce soft tissue trauma and associated complications.

\section{Conflicts of Interest}

The Authors have no conflicts of interest with regard to the work presented.

\section{Authors' Contributions}

All Authors contributed to the design of the study, evaluation of data and writing the manuscript. All Authors have released the manuscript for publication.

\section{References}

1 Shen WJ and Shen YS: Nonsurgical treatment of three-column thoracolumbar junction burst fractures without neurologic deficit. Spine (Phila Pa 1976) 24(4): 412-415, 1999. PMID: 10065527. DOI: 10.1097/00007632-199902150-00024

2 Weninger P, Schultz A and Hertz H: Conservative management of thoracolumbar and lumbar spine compression and burst fractures: functional and radiographic outcomes in 136 cases treated by closed reduction and casting. Arch Orthop Trauma Surg 129(2): 207-219, 2009. PMID: 19009303. DOI: 10.1007/ s00402-008-0780-x

3 Zhao Q, Zhang H, Hao D, Guo H, Wang B and He B: Complications of percutaneous pedicle screw fixation in treating thoracolumbar and lumbar fracture. Medicine (Baltimore) 97(29): e11560, 2018. PMID: 30024554. DOI: 10.1097/MD. 0000000000011560

4 Vanek P, Bradac O, Konopkova R, de Lacy P, Lacman J and Benes V: Treatment of thoracolumbar trauma by short-segment percutaneous transpedicular screw instrumentation: prospective comparative study with a minimum 2-year follow-up. J Neurosurg Spine 20(2): 150-156, 2014. PMID: 24358996. DOI: 10.3171/2013.11.SPINE13479

5 Zhang ZC, Sun TS, Liu Z, Guo YZ and Li LH: [Minimally invasive percutanuous cannulated pedicle screw system fixation for the treatment of thoracolumbar flexion-distraction fracture without neurologic impairment]. Zhongguo Gu Shang 24(10): 802-805, 2011. PMID: 22097123.

6 Alander DH and Cui S: Percutaneous pedicle screw stabilization: Surgical technique, fracture reduction, and review of current spine trauma applications. J Am Acad Orthop Surg 26(7): 231240, 2018. PMID: 29498958. DOI: 10.5435/JAAOS-D-15-00638

7 Wang H, Zhou Y, Li C, Liu J and Xiang L: Comparison of open versus percutaneous pedicle screw fixation using the sextant system in the treatment of traumatic thoracolumbar fractures. Clin Spine Surg 30(3): E239-E246, 2017. PMID: 28323706. DOI: $10.1097 / B S D .0000000000000135$

8 Tinelli M, Töpfer F, Kreinest M, Matschke S, Grützner PA and Suda AJ: Minimally invasive reduction and percutaneous posterior fixation of one-level traumatic thoraco-lumbar and lumbar spine fractures. Eur J Orthop Surg Traumatol 28(8): 1581-1587, 2018. PMID: 29767314. DOI: 10.1007/s00590-0182224-9

9 Loibl M, Korsun M, Reiss J, Gueorguiev B, Nerlich M, Neumann C and Baumann F: Spinal fracture reduction with a minimal-invasive transpedicular Schanz Screw system: clinical and radiological one-year follow-up. Injury 46: S75-S82, 2019. DOI: $10.1016 / \mathrm{S} 0020-1383(15) 30022-\mathrm{X}$

10 Dorr LD, Maheshwari AV, Long WT, Wan Z and Sirianni LE: Early pain relief and function after posterior minimally invasive and conventional total hip arthroplasty. A prospective, randomized, blinded study. J Bone Joint Surg Am 89(6): 11531160, 2007. PMID: 17545416. DOI: $10.2106 / J B J S . F .00940$

11 Rahman M, Summers LE, Richter B, Mimran RI and Jacob RP: Comparison of techniques for decompressive lumbar laminectomy: the minimally invasive versus the "classic" open approach. Minim Invasive Neurosurg 51(2): 100-105, 2008. PMID: 18401823. DOI: 10.1055/s-2007-1022542

12 Hu JC, Gu X, Lipsitz SR, Barry MJ, D’Amico AV, Weinberg AC and Keating NL: Comparative effectiveness of minimally 
invasive $v s$. open radical prostatectomy. JAMA 302(14): 1557 1564, 2009. PMID: 19826025. DOI: 10.1001/jama.2009.1451

13 Mack MJ: Minimally invasive and robotic surgery. JAMA 285(5): 568-572, 2001. PMID: 11176860. DOI: 10.1001/jama. 285.5.568

14 Verlaan JJ, Diekerhof CH, Buskens E, van der Tweel I, Verbout AJ, Dhert WJ and Oner FC: Surgical treatment of traumatic fractures of the thoracic and lumbar spine: a systematic review of the literature on techniques, complications, and outcome. Spine (Phila Pa 1976) 29(7): 803-814, 2004. PMID: 15087804. DOI: $10.1097 / 01 . b r s .0000116990 .31984 . a 9$
15 He Z, Zhang M, Li W, Long Z, Wang L, Li QQ and Lu X: Finite element analysis of an improved correction system for spinal deformity. In Vivo 35(4): 2197-2205, 2021. PMID: 34182497. DOI: 10.21873 /invivo.12491

Received September 23, 2021

Revised October 15, 2021

Accepted November 16, 2021 